\title{
$\mu$-LINDELÖFNESS IN TERMS OF A HEREDITARY CLASS
}

\author{
ABDO QAHIS, HEYAM HUSSAIN ALJARRAH, AND TAKASHI NOIRI
}

\begin{abstract}
A hereditary class on a set $X$ is a nonempty collection of subsets of $X$ closed under the hereditary property. In this paper, we define and study the notion of Lindelöfness in generalized topological spaces with respect to a hereditary class called, $\mu \mathcal{H}$-Lindelöf spaces and discuss their properties.
\end{abstract}

\section{INTRODUCTION}

The Lindelöfness is an important and interesting concept in general topology. This paper will not only study general topology, but also other areas of mathematics. During the last few years several authors have been working to formulate weak notions of open sets. In terms of these open sets those authors have extended and generalized the concept of Lindelöfness. The purpose of the present paper is to introduce and investigate the concept of $\mu$-Lindelöfness by using the notions of generalized topology and hereditary class which are introduced by Császár in [1] and [2], respectively. Also some properties of $\mu \mathcal{H}$-Lindelöfness spaces are obtained. The strategy of using generalized topologies and hereditary classes to extend classical topological concepts have been used by many authors such as $[2,9,12,17]$, among others.

\section{Preliminaries}

Let $X$ be a non-empty set and $2^{X}$ denote the power set of $X$. We call a class $\mu \subseteq 2^{X}$ a generalized topology [1] (briefly, GT) if $\phi \in \mu$ and arbitrary union of elements of $\mu$ belongs to $\mu$. A set $X$ with a GT is called a generalized topological space (briefly, GTS) and is denoted by $(X, \mu)$. For a GTS $(X, \mu)$, the elements of $\mu$ are called $\mu$-open sets and the complement of $\mu$-open sets are called $\mu$-closed sets. For $A \subseteq X$, we denote by $c_{\mu}(A)$ the intersection of all $\mu$-closed sets containing $A$, i.e., the smallest $\mu$-closed set containing $A$ and by $i_{\mu}(A)$ the union of all $\mu$-open sets contained in $A$, i.e., the largest $\mu$-open set contained in $A$ (see $[1,3]$ ). Let $A \subset X$. A family $\mathcal{C}$ of subsets of $X$ is called a $\mu$-covering of $A$ if $\mathcal{C}$ is a covering of $A$ by $\mu$-open sets [8]. A subset $A$ of $X$ is said to be $\mu$-Lindelöf relative to $X$ if for every $\mu$-covering $\left\{U_{\lambda}: \lambda \in \Lambda\right\}$ of $A$ there exists a countable subfamily

MISSOURI J. OF MATH. SCI., SPRING 2016 


\section{A. QAHIS, H. H. ALJARRAH, T. NOIRI}

$\left\{U_{\lambda}: \lambda \in \Lambda_{0}\right\}$ such that it covers $A . X$ is said to be $\mu$-Lindelöf if $X$ is $\mu$-Lindelöf as a subset [15].

A nonempty family $\mathcal{H}$ of subsets of $X$ is called a hereditary class [2] if $A \in \mathcal{H}$ and $B \subset A$ imply that $B \in \mathcal{H}$. Given a generalized topological space $(X, \mu)$ with a hereditary class $\mathcal{H}$, for a subset $A$ of $X$, the generalized local function of $A$ with respect to $\mathcal{H}$ and $\mu$ [2] is defined as follows: $A^{\star}=\{x \in$ $X: U \cap A \notin \mathcal{H} \quad$ for all $\left.U \in \mu_{x}\right\}$, where $\mu_{x}=\{U: x \in U$ and $U \in \mu\}$; and the following are defined: $c_{\mu}^{\star}(A)=A \cup A^{\star}$ and the family $\mu^{\star}=\{A \subset$ $\left.X: X \backslash A=c_{\mu}^{\star}(X \backslash A)\right\}$. If the hereditary class $\mathcal{H}$ satisfies the additional condition: if $A, B \in \mathcal{H}$ implies $A \cup B \in \mathcal{H}$, then $\mathcal{H}$ is called an ideal on $X[10]$. We call $(X, \mu, \mathcal{H})$ a hereditary generalized topological space and briefly we denote it by HGTS. If there is no confusion, we simply write $A^{\star}$ instead of $A^{\star}(\mathcal{H}, \mu)$. It is clear that a subset $A$ is $\mu^{\star}$-closed if and only if $A^{\star} \subset A$.

Definition 2.1. [1] Let $(X, \mu)$ and $(Y, \nu)$ be two GTSs, then a function $f$ : $(X, \mu) \rightarrow(Y, \nu)$ is said to be $(\mu, \nu)$-continuous if $U \in \nu$ implies $f^{-1}(U) \in \mu$.

Definition 2.2. [16] A function $f:(X, \mu) \rightarrow(Y, \nu)$ is $(\mu, \nu)$-open (or $\mu$-open) if $U \in \mu$ implies $f(U) \in \nu$.

Definition 2.3. Let $(X, \mu)$ be a GTS. Then a subset $A$ of $X$ is said to be $\mu$-dense [6] if $c_{\mu}(A)=X$. The space $(X, \mu)$ is said to be $\mu$-submaximal [7] if every $\mu$-dense subset is $\mu$-open in $X$.

Definition 2.4. Let $(X, \mu)$ be a GTS. Then a subset $A$ of $X$ is called a $\mu$ generalized closed set (in short, a $\mu$ g-closed set) $[13]$ if $c_{\mu}(A) \subseteq U$ whenever $A \subseteq U$ where $U$ is $\mu$-open in $X$. The complement of a $\mu g$-closed set is called a $\mu g$-open set.

Definition 2.5. [4] A GTS $(X, \mu)$ is said to be $\mu$-extremally disconnected if the $\mu$-closure of every $\mu$-open set is $\mu$-open.

Theorem 2.6. [2] Let $(X, \mu)$ be a GTS and $\mathcal{H}$ be a hereditary class on $X$ and $A$ a subset of $X$, then $A^{\star} \subset c_{\mu}(A)$.

Theorem 2.7. [2] Let $(X, \mu)$ be a GTS, $\mathcal{H}$ a hereditary class on $X$ and $A$ be a subset of $X$. If $A$ is $\mu^{\star}$-open, then for each $x \in A$ there exist $U \in \mu_{x}$ and $H \in \mathcal{H}$ such that $x \in U \backslash H \subset A$.

\section{3. $\mu \mathcal{H}$-LindelÖF SPACES}

Definition 3.1. Let $(X, \mu)$ be a GTS and $\mathcal{H}$ be a hereditary class on $X$. A HGTS $(X, \mu, \mathcal{H})$ is said to be $\mu \mathcal{H}$-Lindelöf or $\mu$-Lindelöf with respect to a hereditary class $\mathcal{H}$ if for every $\mu$-covering $\left\{U_{\lambda}: \lambda \in \Lambda\right\}$ of $X$ there exists a countable subset $\Lambda_{0}$ of $\Lambda$ such that $X \backslash \cup\left\{U_{\lambda}: \lambda \in \Lambda_{0}\right\} \in \mathcal{H}$. 


\section{$\mu$-LINDELÖFNESS IN TERMS OF A HEREDITARY CLASS}

The following theorem gives a characterization of $\mu \mathcal{H}$-Lindelöfness.

Theorem 3.2. The following are equivalent for a $\operatorname{HGTS}(X, \mu, \mathcal{H})$ :

(1) $(X, \mu, \mathcal{H})$ is $\mu \mathcal{H}$-Lindelöf;

(2) For any family $\left\{F_{\lambda}: \lambda \in \Lambda\right\}$ of $\mu$-closed sets of $X$ such that $\cap\left\{F_{\lambda}\right.$ : $\lambda \in \Lambda\}=\phi$, there exists a countable subset $\Lambda_{0}$ of $\Lambda$ such that $\cap\left\{F_{\lambda}: \lambda \in \Lambda_{0}\right\} \in \mathcal{H}$.

Proof. (1) $\Rightarrow$ (2): Let $\left\{F_{\lambda}: \lambda \in \Lambda\right\}$ be a family of $\mu$-closed sets of $X$ such that $\cap\left\{F_{\lambda}: \lambda \in \Lambda\right\}=\phi$. Then $\left\{X \backslash F_{\lambda}: \lambda \in \Lambda\right\}$ is a $\mu$-covering of $X$. By (1) $(X, \mu, \mathcal{H})$ is $\mu \mathcal{H}$-Lindelöf, there exists a countable subset $\Lambda_{0}$ of $\Lambda$ such that $X \backslash \cup\left\{X \backslash F_{\lambda}: \lambda \in \Lambda_{0}\right\} \in \mathcal{H}$. This implies that $\cap\left\{F_{\lambda}: \lambda \in \Lambda_{0}\right\} \in \mathcal{H}$. $(2) \Rightarrow(1)$ : Let $\left\{U_{\lambda}: \lambda \in \Lambda\right\}$ be any $\mu$-covering of $X$, then $\left\{X \backslash U_{\lambda}: \lambda \in \Lambda\right\}$ is a family of $\mu$-closed sets and $\cap\left\{X \backslash U_{\lambda}: \lambda \in \Lambda\right\}=\phi$. Hence, there exists a countable subset $\Lambda_{0}$ of $\Lambda$ such that $\cap\left\{X \backslash U_{\lambda}: \lambda \in \Lambda_{0}\right\} \in \mathcal{H}$. This implies that $\cap\left\{X \backslash U_{\lambda}: \lambda \in \Lambda_{0}\right\}=X \backslash \cup\left\{U_{\lambda}: \lambda \in \Lambda_{0}\right\} \in \mathcal{H}$. This shows that $(X, \mu, \mathcal{H})$ is $\mu \mathcal{H}$-Lindelöf.

Theorem 3.3. Let $(X, \mu)$ be a GTS and $\mathcal{H}$ be a hereditary class on $X$. Then, the following statements hold.

(1) If a $\operatorname{HGTS}\left(X, \mu^{*}, \mathcal{H}\right)$ is $\mu^{*} \mathcal{H}$-Lindelöf, then $(X, \mu, \mathcal{H})$ is $\mu \mathcal{H}$ Lindelöf.

(2) If a $\operatorname{HGTS}(X, \mu, \mathcal{H})$ is $\mu \mathcal{H}$-Lindelöf and the class $\mathcal{H}$ is closed under countable union, then the $\operatorname{HGTS}\left(X, \mu^{*}, \mathcal{H}\right)$ is $\mu^{*} \mathcal{H}$-Lindelöf.

Proof. (1): The proof follows directly from the fact that every $\mu$-closed set is $\mu^{*}$-closed set.

(2): Suppose that $\mathcal{H}$ is closed under countable union and $X$ is $\mu \mathcal{H}$-Lindelöf. Given $\left\{U_{\lambda}: \lambda \in \Lambda\right\}$ a $\mu^{\star}$-covering of $X$, then for each $x \in X, x \in U_{\lambda_{x}}$ for some $\lambda_{x} \in \Lambda$. By Theorem 2.6, there exist $V_{\lambda_{x}} \in \mu_{x}$ and $H_{\lambda_{x}} \in \mathcal{H}$ such that $x \in V_{\lambda_{x}} \backslash H_{\lambda_{x}} \subset U_{\lambda_{x}}$. Since the family $\left\{V_{\lambda_{x}}: \lambda_{x} \in \Lambda\right\}$ is a $\mu$-covering of $X$, it follows that there exists a countable subset $\Lambda_{0}$ of $\Lambda$ such that $H=X \backslash \cup\left\{V_{\lambda_{x}}: \lambda_{x} \in \Lambda_{0}\right\} \in \mathcal{H}$. Since $\mathcal{H}$ is closed under countable union, then $\cup\left\{H_{\lambda_{x}}: \lambda_{x} \in \Lambda_{0}\right\} \in \mathcal{H}$. Hence, $H \cup\left[\cup\left\{H_{\lambda_{x}}: \lambda_{x} \in \Lambda_{0}\right\}\right] \in \mathcal{H}$. Observe that $X \backslash \cup\left\{U_{\lambda}: \lambda \in \Lambda_{0}\right\} \subset H \cup\left[\cup\left\{H_{\lambda_{x}}: \lambda_{x} \in \Lambda_{0}\right\}\right] \in \mathcal{H}$. By the heredity property of the class $H$ we have $X \backslash \cup\left\{U_{\lambda}: \lambda \in \Lambda_{0}\right\} \in \mathcal{H}$ and therefore, $\left(X, \mu^{*}, \mathcal{H}\right)$ is $\mu^{*} \mathcal{H}$-Lindelöf.

Remark 3.4. To show that the assumption $\mathcal{H}$ is closed under countable union in (2) of Theorem 3.3 is required in the hypotheses, we consider the following example.

Example 3.5. Let $\mathbb{R}$ be the set of real numbers and $\mu$ be the generalized topology defined as

$$
\mu=\{A \subset \mathbb{R}: A \text { is an uncountable set }\} \cup\{\phi\} .
$$

MISSOURI J. OF MATH. SCI., SPRING 2016 


\section{A. QAHIS, H. H. ALJARRAH, T. NOIRI}

The hereditary class on $\mathbb{R}$ is defined as

$$
\mathcal{H}=\{\mathbb{R} \backslash A: A \in \mu\} .
$$

Observe that $\mathcal{H}$ is not an ideal. To show that $\mathbb{R}$ is $\mu \mathcal{H}$-Lindelöf, let $\left\{U_{\lambda}\right.$ : $\lambda \in \Lambda\}$ be any $\mu$-covering of $\mathbb{R}$. Any countable subfamily $\left\{U_{\lambda}: \lambda \in \Lambda_{0}\right\}$, where $\Lambda_{0} \subset \Lambda$. We obtain, $\mathbb{R} \backslash \cup\left\{U_{\lambda}: \lambda \in \Lambda_{0}\right\} \subset \mathbb{R} \backslash U_{\lambda} \in \mathcal{H}$. It follows that $\mathbb{R}$ is $\mu \mathcal{H}$-Lindelöf. Observe that for each $x \in \mathbb{R}$ we have $(\mathbb{R} \backslash$ $\{x\})^{\star} \subset \mathbb{R} \backslash\{x\}$. Thus, $\{x\}$ is $\mu^{\star}$-open for each $x \in \mathbb{R}$. It then follows that $\{\{x\}: x \in \mathbb{R}\}$ is a $\mu^{*}$-covering of $\mathbb{R}$. Suppose now that there exist countable $x_{1}, x_{2}, \ldots, x_{n}, \ldots \in \mathbb{R}$ such that $\mathbb{R} \backslash \bigcup_{i=1}^{\infty}\left\{x_{i}\right\} \in \mathcal{H}$. But this is impossible. Therefore, $\mathbb{R}$ is not $\mu^{*} \mathcal{H}$-Lindelöf.

Given a generalized topological space $(X, \mu)$, we denote by $\mathcal{H}_{c}$ the hereditary class of countable subsets of $X$. The following proposition is obvious and thus the proof are omitted.

Proposition 3.6. Let $(X, \mu)$ be a GTS and $\mathcal{H}$ be a hereditary class on $X$. Then the following statements are equivalent:

(1) $(X, \mu)$ is $\mu$-Lindelöf;

(2) $\left(X, \mu, \mathcal{H}_{c}\right)$ is $\mu \mathcal{H}_{c}$-Lindelöf;

(3) $(X, \mu,\{\phi\})$ is $\mu\{\phi\}-$ Lindelöf.

Corollary 3.7. If the HGTS $\left(X, \mu, \mathcal{H}_{c}\right)$ is $\mu \mathcal{H}_{c}$-compact, then $(X, \mu)$ is $\mu \mathcal{H}_{c}$-Lindelöf.

Proof. Let $\left\{U_{\lambda}: \lambda \in \Lambda\right\}$ be a $\mu$-covering of $X$. Since $\left(X, \mu, \mathcal{H}_{c}\right)$ is $\mu \mathcal{H}_{c^{-}}$ compact, there exists a finite subset $\Lambda_{0}$ of $\Lambda$ such that $X \backslash\left\{U_{\lambda}: \lambda \in\right.$ $\left.\Lambda_{0}\right\} \in \mathcal{H}_{C}$ This shows that $X$ has a countable subcover and the proof is completed.

Proposition 3.8. Let $\mathcal{H}_{1}$ and $\mathcal{H}_{2}$ be two hereditary classes on a GTS $(X, \mu)$ with $\mathcal{H}_{1} \subseteq \mathcal{H}_{2}$. If $\left(X, \mu, \mathcal{H}_{1}\right)$ is $\mu \mathcal{H}_{1}$-Lindelöf, then $\left(X, \mu, \mathcal{H}_{2}\right)$ is $\mu \mathcal{H}_{2}$-Lindelöf.

Proof. Suppose that $\left(X, \mu, \mathcal{H}_{1}\right)$ is $\mu \mathcal{H}_{1}$-Lindelöf. Let $\left\{U_{\lambda}: \lambda \in \Lambda\right\}$ be any $\mu$-covering of $X$. There exits a countable subset $\Lambda_{0}$ of $\Lambda$ such that $X \backslash \cup\left\{U_{\lambda}: \lambda \in \Lambda_{0}\right\} \in \mathcal{H}_{1} \subset \mathcal{H}_{2}$. This implies that $X \backslash \cup\left\{U_{\lambda}: \lambda \in \Lambda_{0}\right\} \in \mathcal{H}_{2}$. Hence, $X$ is $\mu \mathcal{H}_{2}$-Lindelöf.

Remark 3.9. The intersection of any two hereditary classes on a nonempty set $X$ is a heredity class. To prove this, let $\mathcal{H}_{1}$ and $\mathcal{H}_{2}$ be any two hereditary classes on $X$. If $A \in \mathcal{H}_{1} \cap \mathcal{H}_{2}$ and $B \subset A$ then $B \subset A \in \mathcal{H}_{1}$ and $B \subset A \in \mathcal{H}_{2}$. By the hypotheses on $\mathcal{H}_{1}$ and $\mathcal{H}_{2}$ we have $B \in \mathcal{H}_{1}$ and $B \in \mathcal{H}_{2}$. It then follows that $B \in \mathcal{H}_{1} \cap \mathcal{H}_{2}$. 


\section{$\mu$-LINDELÖFNESS IN TERMS OF A HEREDITARY CLASS}

Corollary 3.10. If $\left(X, \tau, \mathcal{H}_{1} \cap \mathcal{H}_{2}\right)$ is $\mu\left(\mathcal{H}_{1} \cap \mathcal{H}_{2}\right)$ - Lindelöf, then $\left(X, \mu, \mathcal{H}_{1}\right)$ is $\mu \mathcal{H}_{1}$-Lindelöf and $\left(X, \mu, \mathcal{H}_{2}\right)$ is $\mu \mathcal{H}_{2}$-Lindelöf.

A subset $A$ of GTS is said to be $\mu$-semi-open if $A \subset c_{\mu}\left(i_{\mu}(A)\right)$ and we denote by $\sigma(\mu)$ the class of all $\mu$-semi-open sets [3].

Definition 3.11. A HGTS $(X, \mu, \mathcal{H})$ is said to be $\mu \mathcal{H}$-semi-Lindelöf if for every $\mu$-semi-open cover $\left\{U_{\lambda}: \lambda \in \Lambda\right\}$ of $X$ there exists a countable subset $\Lambda_{0}$ of $\Lambda$ such that $X \backslash \cup\left\{U_{\lambda}: \lambda \in \Lambda_{0}\right\} \in \mathcal{H}$.

Proposition 3.12. Every $\mu \mathcal{H}$-semi-Lindelöf space is $\mu \mathcal{H}$-Lindelöf.

Proof. The proof is obvious since every $\mu$-open set is $\mu$-semi-open.

Proposition 3.13. If $(X, \mu)$ is $\mu$-submaximal and extremally disconnected, then $\mu \mathcal{H}$-Lindelöf and $\mu \mathcal{H}$-semi-Lindelöf are equivalent.

Proof. The proof comes immediately from the fact that in the extremely disconnected $\mu$-submaximal space $\mu=\sigma(\mu)$.

The following lemma is very useful in studying the preservation of $\mu \mathcal{H}$ Lindelöfness by certain classes of functions.

Lemma 3.14. [5] Let $f:(X, \mu, \mathcal{H}) \rightarrow(Y, \nu)$ be a function. If $\mathcal{H}$ is a hereditary class on $X$, then $f(\mathcal{H})=\{f(E): E \in \mathcal{H}\}$ is a hereditary class on $Y$.

Theorem 3.15. If $f:(X, \mu, \mathcal{H}) \rightarrow(Y, \nu)$ is a $(\mu, \nu)$-continuous surjection and $(X, \mu, \mathcal{H})$ is $\mu \mathcal{H}$-Lindelöf, then $(Y, \nu, f(\mathcal{H}))$ is $\nu f(\mathcal{H})$-Lindelöf.

Proof. Let $\left\{V_{\lambda}: \lambda \in \Lambda\right\}$ be a $\nu$-covering of $Y$. Then $\left\{f^{-1}\left(V_{\lambda}\right): \lambda \in \Lambda\right\}$ is a $\mu$-covering of $X$ and hence, there exists a countable subset $\Lambda_{0}$ of $\Lambda$ such that $X \backslash \cup\left\{f^{-1}\left(V_{\lambda}\right): \lambda \in \Lambda_{0}\right\} \in \mathcal{H}$. Since $f$ is a surjective function, by Lemma 3.14 we have $Y \backslash \cup\left\{V_{\lambda}: \lambda \in \Lambda_{0}\right\} \subset f\left(X \backslash \cup\left\{f^{-1}\left(V_{\lambda}\right): \lambda \in \Lambda_{0}\right\}\right) \in f(\mathcal{H})$ implying thereby that $Y$ is $\nu f(\mathcal{H})$-Lindelöf.

By taking $\mathcal{H}=\{\phi\}$ in the above theorem, we get the well-known result that $\mu$-Lindelöf is preserved by $(\mu, \nu)$-continuous surjections. We also have the following results concerning the pre-images.

Corollary 3.16. If $f:(X, \mu) \rightarrow(Y, \nu, \mathcal{H})$ is a $\mu$-open bijection and $(Y, \nu, \mathcal{H})$ is $\nu \mathcal{H}$-Lindelöf, then $\left(X, \mu, f^{-1}(\mathcal{H})\right)$ is $\mu f^{-1}(\mathcal{H})$-Lindelöf.

Proof. Since $f:(X, \mu) \rightarrow(Y, \nu, \mathcal{H})$ is a $\mu$-open bijection, $f^{-1}:(Y, \nu, \mathcal{H}) \rightarrow$ $(X, \mu)$ is a $(\nu, \mu)$-continuous surjection. Since $(Y, \nu, \mathcal{H})$ is $\nu \mathcal{H}$ - Lindelöf, by Theorem 3.15 we obtain $\left(X, \mu, f^{-1}(\mathcal{H})\right)$ is $\mu f^{-1}(\mathcal{H})$-Lindelöf. 


\section{Sets $\mu \mathcal{H}$-Lindelöf Relative to a Space}

Definition 4.1. Let $(X, \mu)$ be a GTS and $\mathcal{H}$ be a hereditary class on $X$. $A$ subset $A$ of $X$ is said to be $\mu \mathcal{H}$-Lindelöf or $\mu$-Lindelöf with respect to $(X, \mu, \mathcal{H})$ if for every $\mu$-covering $\left\{U_{\lambda}: \lambda \in \Lambda\right\}$ of $A$ there exists a countable subset $\Lambda_{0}$ of $\Lambda$ such that $A \backslash \cup\left\{U_{\lambda}: \lambda \in \Lambda_{0}\right\} \in \mathcal{H}$.

Definition 4.2. A subset $A$ of $G T S(X, \mu)$ is said to be $\omega$ - $\mu$-open if for each $x \in A$, there exists $U_{x} \in \mu$ containing $x$ such that $U_{x} \backslash A$ is a countable set.

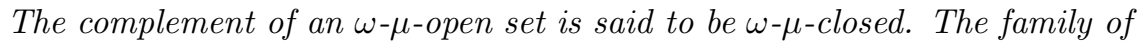
all $\omega$ - $\mu$-open sets of $(X, \mu)$ is denoted by $\mu_{\omega}$.

Lemma 4.3. For any $G T S(X, \mu)$, the family $\mu_{\omega}$ is $G T$.

Proof. It is obvious that $\phi, X \in \mu_{\omega}$. Let $\left\{A_{\lambda}: \lambda \in \Lambda\right\}$ be any subfamily of $\mu_{\omega}$. Then for each $x \in \bigcup_{\lambda \in \Lambda} A_{\lambda}$, there exists $\lambda_{0} \in \Lambda$ such that $x \in A_{\lambda_{0}}$. Since $A_{\lambda_{0}} \in \mu_{\omega}$, there exists $U_{x} \in \mu$ containing $x$ such that $U_{x} \backslash A_{\lambda_{0}}$ is a countable set. Since $U_{x} \backslash\left(\bigcup_{\lambda \in \Lambda} A_{\lambda}\right) \subset U_{x} \backslash A_{\lambda_{0}}, U_{x} \backslash\left(\bigcup_{\lambda \in \Lambda} A_{\lambda}\right)$ is a countable set. Therefore, $\bigcup_{\lambda \in \Lambda} A_{\lambda} \in \mu_{\omega}$. This shows that $\left(X, \mu_{\omega}\right)$ is GTS.

Theorem 4.4. A subset $A$ of a $\operatorname{HGTS}(X, \mu, \mathcal{H})$ is $\mu \mathcal{H}$-Lindelöf relative to $\mu$ if and only if $A$ is $\mu_{\omega} \mathcal{H}$-Lindelöf relative to $\mu_{\omega}$.

Proof. Necessity. Suppose that a subset $A$ of a $\operatorname{HGTS}(X, \mu, \mathcal{H})$ is $\mu \mathcal{H}$ Lindelöf relative to $\mu$. Let $\left\{U_{\lambda}: \lambda \in \Lambda\right\}$ be any $\mu_{\omega}$-covering of $A$. For each $x \in A$ there exists $\lambda(x) \in \Lambda$ such that $x \in U_{\lambda(x)}$. Since $U_{\lambda(x)}$ is $\mu_{\omega}$-open, there exists a $\mu$-open set $V_{\lambda(x)}$ such that $x \in V_{\lambda(x)}$ and $V_{\lambda(x)} \backslash$ $U_{\lambda(x)}$ is countable. The collection $\left\{V_{\lambda(x)}: x \in A\right\}$ is a $\mu$-covering of $A$. Since $A$ is $\mu \mathcal{H}$-Lindelöf relative to $\mu$, there exists a countable subset, $\lambda\left(x_{1}\right), \lambda\left(x_{2}\right), \ldots, \lambda\left(x_{n}\right), \ldots$ such that $A \backslash \cup\left\{V_{\lambda\left(x_{i}\right)}: i \in \mathbb{N}\right\} \in \mathcal{H}$. On the other hand, we have

$$
A \backslash \cup_{i \in \mathbb{N}}\left\{\left(V_{\lambda\left(x_{i}\right)} \backslash U_{\lambda\left(x_{i}\right)}\right) \cup U_{\lambda\left(x_{i}\right)}\right\} \subset A \backslash \cup\left\{V_{\lambda\left(x_{i}\right)}: i \in \mathbb{N}\right\}
$$

and hence,

$$
\left.A \backslash\left[\cup_{i \in \mathbb{N}}\left(V_{\lambda\left(x_{i}\right)} \backslash U_{\lambda\left(x_{i}\right)}\right) \cap A\right) \cup\left(\cup_{i \in \mathbb{N}} U_{\lambda\left(x_{i}\right)}\right)\right] \subset A \backslash \cup\left\{V_{\lambda\left(x_{i}\right)}: i \in \mathbb{N}\right\} .
$$

For each $\lambda\left(x_{i}\right)$, the set $\left(V_{\lambda\left(x_{i}\right)} \backslash U_{\lambda\left(x_{i}\right)}\right) \cap A$ is a countable set and there exists a countable subset $\Lambda_{\lambda\left(x_{i}\right)}$ of $\Lambda$ such that $\left(V_{\lambda\left(x_{i}\right)} \backslash U_{\lambda\left(x_{i}\right)}\right) \cap A \subseteq$ 
$\cup\left\{U_{\lambda}: \lambda \in \Lambda_{\lambda\left(x_{i}\right)}\right\}$. Therefore, we have

$$
\begin{aligned}
A & \backslash\left[\left(\cup_{i \in \mathbb{N}}\left(\cup\left\{U_{\lambda}: \lambda \in \Lambda_{\lambda\left(x_{i}\right)}\right\}\right)\right) \cup\left(\cup_{i \in \mathbb{N}} U_{\lambda\left(x_{i}\right)}\right)\right] \\
& \subset A \backslash\left[\left(\cup_{i \in \mathbb{N}}\left(V_{\lambda\left(x_{i}\right)} \backslash U_{\lambda\left(x_{i}\right)}\right) \cap A\right) \cup\left(\cup_{i \in \mathbb{N}} U_{\lambda\left(x_{i}\right)}\right)\right] \\
& \subset A \backslash \cup\left\{V_{\lambda\left(x_{i}\right)}: i \in \mathbb{N}\right\} \in \mathcal{H} .
\end{aligned}
$$

By the hereditary property of the class $\mathcal{H}$, we have

$$
A \backslash\left[\left(\cup_{i \in \mathbb{N}}\left(\cup\left\{U_{\lambda}: \lambda \in \Gamma_{\lambda\left(x_{i}\right)}\right\}\right)\right) \cup\left(\cup_{i \in \mathbb{N}} U_{\lambda\left(x_{i}\right)}\right)\right] \in \mathcal{H} .
$$

Sufficiency. Since $\mu \subset \mu_{\omega}$ the proof is obvious.

Corollary 4.5. A HGTS $(X, \mu, \mathcal{H})$ is $\mu \mathcal{H}$-Lindelöf if and only if the HGTS $\left(X, \mu_{\omega}, \mathcal{H}\right)$ is $\mu_{\omega} \mathcal{H}$-Lindelöf.

By taking $\mathcal{H}=\{\phi\}$ in the Corollary 4.5, we obtain the following result established in Theorem 4.4.

Theorem 4.6. A GTS $(X, \mu)$ is $\mu$-Lindelöf if and only if $\left(X, \mu_{\omega}\right)$ is $\mu_{\omega}$ Lindelöf.

Definition 4.7. [5] Let $(X, \mu)$ be a GTS and $\mathcal{H}$ be a hereditary class on $X$. A subset $A$ of $X$ is said to be $\mu \mathcal{H}$-compact if for every $\mu$-covering $\left\{U_{\lambda}: \lambda \in \Lambda\right\}$ of $A$ there exists a finite subcollection $\left\{U_{\lambda}: \lambda \in \Lambda_{0}\right\}$ such that $A \backslash \cup\left\{U_{\lambda}: \lambda \in \Lambda_{0}\right\} \in \mathcal{H} . X$ is said to be a $\mu \mathcal{H}$-compact space if $X$ is $\mu \mathcal{H}$-compact as a subset.

A $\operatorname{GTS}(X, \mu)$ is said to be $\mu$-Hausdroff [14] for each pair of distinct points $x$ and $y$ in $X$, there exist $\mu$-open sets $U_{x}$ and $V_{y}$ containing $x$ and $y$, respectively, such that $U \cap V=\phi$.

Lemma 4.8. $x \notin A^{*}$ if and only if $\left(U_{x} \backslash H\right) \cap A=\phi$, where $U_{x} \in \mu(x)$ and $H \in \mathcal{H}$.

Proof. Let $x \notin A^{*}$. Then there exist $U_{x} \in \mu(x)$ such that $A \cap U_{x}=H \in \mathcal{H}$. It follows that $\left(U_{x} \backslash H\right) \cap A=\phi$. Conversely, suppose that $\left(U_{x} \backslash H\right) \cap A=\phi$ for some $U_{x} \in \mu(x)$ and $H \in \mathcal{H}$. Then $U_{x} \cap(X \backslash H) \cap A=\left(A \cap U_{x}\right) \cap(X \backslash H)=$ $\phi$. This implies that $\left(A \cap U_{x}\right) \subset H \in \mathcal{H}$. Hence, $x \notin A^{*}$.

Theorem 4.9. Every $\mu \mathcal{H}$-compact subset of a $\mu$-Hausdorff $H G T S(X, \mu, \mathcal{H})$ is $\mu^{*}$-closed.

Proof. Let $A$ be a $\mu \mathcal{H}$-compact subset of a $\mu$-Hausdroff $\operatorname{HGTS}(X, \mu, \mathcal{H})$. Let $x \notin A$ then $x \in X \backslash A$. For each $y \in A$, there exist two $\mu$-open sets $U_{y}$ and $V_{y}$ containing $x$ and $y$, respectively, such that $U_{y} \cap V_{y}=\phi$. Note that $x \notin c_{\mu}\left(V_{y}\right)$. Then $\left\{V_{y}: y \in A\right\}$ is a $\mu$-covering of $A$ which is $\mu \mathcal{H}$-compact. 
Therefore, there exists a finite subset $\Lambda_{0}$ of $A$ such that $A \backslash \cup\left\{V_{y}: y \in \Lambda_{0}\right\} \in$ $\mathcal{H}$. Now $x \notin c_{\mu}\left(V_{y}\right)$ for each $y \in A$ implies $x \notin \bigcup_{y \in \Lambda_{0}} c_{\mu}\left(V_{y}\right)=c_{\mu}\left(\bigcup_{y \in \Lambda_{0}} V_{y}\right)$. Let $U=X \backslash c_{\mu}\left(\bigcup_{y \in \Lambda_{0}} V_{y}\right)$ and let $H=A \backslash c_{\mu}\left(\bigcup_{y \in \Lambda_{0}}^{y \in \Lambda_{0}} V_{y}\right) \subset A \backslash \bigcup_{y \in \Lambda_{0}} V_{y}=H_{1}$, where $H_{1} \in \mathcal{H}$. Since $U \in \mu_{x}$ and $H \in \mathcal{H}$, by Theorem $2.7 U \backslash H$ is a $\mu^{*}$-open set containing $x$ and $(U \backslash H) \cap A=\emptyset$. This implies that $x \notin A^{*}$ (by Lemma 4.8). Hence, $A^{*} \subset A$, so $A$ is $\mu^{*}$-closed.

Theorem 4.10. Let $(X, \mu)$ be a GTS and $\mathcal{H}$ be an ideal on $X$, then a finite union of sets which are $\mu \mathcal{H}$ - Lindelöf relative to a space $(X, \mu, \mathcal{H})$ is a $\mu \mathcal{H}$-Lindelöf relative to $X$.

Proof. Let $A_{1}$ and $A_{2}$ be two subsets which are $\mu \mathcal{H}$-Lindelöf relative to $X$ and let $A=A_{1} \cup A_{2}$. Let $\left\{U_{\lambda}: \lambda \in \Lambda\right\}$ be a $\mu$-covering of $A$. Hence, $\left\{U_{\lambda}: \lambda \in \Lambda\right\}$ is a $\mu$-covering of $A_{1}$ and $A_{2}$. Since $A_{1}$ and $A_{2}$ are $\mu \mathcal{H}$-Lindelöf relative to $X$, there exist finite subfamily $\left\{H_{1}, H_{2}\right\} \subset \mathcal{H}$ and countable subsets $\Lambda_{0}$ and $\Lambda_{1}$ of $\Lambda$ such that $A_{1} \backslash \cup\left\{U_{\lambda_{i}}: \lambda_{i} \in \Lambda_{0}\right\}=H_{1}$ and $A_{2} \backslash$ $\cup\left\{U_{\lambda_{k}}: \lambda_{k} \in \Lambda_{1}\right\}=H_{2}$. Now we have

$$
\begin{aligned}
A & =A_{1} \cup A_{2} \\
& \subset\left(\cup\left\{U_{\lambda_{i}}: \lambda_{i} \in \Lambda_{0}\right\}\right) \cup\left(\cup\left\{U_{\lambda_{k}}: \lambda_{k} \in \Lambda_{1}\right\}\right) \cup\left(H_{1} \cup H_{2}\right) .
\end{aligned}
$$

This implies $\left(A_{1} \cup A_{2}\right) \backslash\left(\cup\left\{U_{\lambda_{i}}: \lambda_{i} \in \Lambda_{0}\right\}\right) \cup\left(\cup\left\{U_{\lambda_{k}}: \lambda_{k} \in \Lambda_{1}\right\}\right) \subset$ $H_{1} \cup H_{2} \in \mathcal{H}$. Therefore, $A=A_{1} \cup A_{2}$ is $\mu \mathcal{H}$ - Lindelöf relative to $X$. This proves that the union of two $\mu \mathcal{H}$-Lindelöf sets is $\mu \mathcal{H}$-Lindelöf. For finite unions, the proof proceeds by induction on the number of sets.

Remark 4.11. If the class $\mathcal{H}$ is not an ideal then the union of finite subsets which are $\mu \mathcal{H}$-Lindelöf relative to $X$ is not $\mu \mathcal{H}$-Lindelöf relative to $X$.

Let $(X, \mu, \mathcal{H})$ be a HGTS and let $A \subseteq X, A \neq \phi$. We denote by $\mathcal{H}_{A}$ the collection $\{H \cap A: H \in \mathcal{H}\}$ and by $\left(A, \mu_{A}\right)$ the subspace of $(X, \mu)$ on $A$. It is clear that the collection $\mu_{A}$ is a generalized topology on $A$ and the collection $\mathcal{H}_{A}$ is a hereditary class of subsets in $A$. Then we have the following theorem.

Theorem 4.12. Let $(X, \mu, \mathcal{H})$ be a $\mu \mathcal{H}$-Lindelöf HGTS and $A$ be a $\mu$-closed subset of $X$. Then $\left(A, \mu_{A}, \mathcal{H}_{A}\right)$ is $\mu_{A} \mathcal{H}_{A}$-Lindelöf.

Proof. Let $\left\{U_{\lambda} \cap A: U_{\lambda} \in \mu, \lambda \in \Lambda\right\}$ be a $\mu_{A}$-covering of $A$. Then $\left\{U_{\lambda}\right.$ : $\left.U_{\lambda} \in \mu, \lambda \in \Lambda\right\} \cup\{X \backslash A\}$ is a $\mu$-covering of $X$ and hence, there exists a countable subset $\Lambda_{0}$ of $\Lambda$ such that $X \backslash\left[\cup\left\{U_{\lambda}: \lambda \in \Lambda_{0}\right\} \cup(X \backslash A)\right]=H \in \mathcal{H}$. 


\section{$\mu$-LINDELÖFNESS IN TERMS OF A HEREDITARY CLASS}

Now, we have

$$
\begin{aligned}
A \cap H & =A \cap\left(X \backslash\left[\cup\left\{U_{\lambda}: \lambda \in \Lambda_{0}\right\} \cup(X \backslash A)\right]\right) \\
& =A \cap\left(X \backslash \cup\left\{U_{\lambda}: \lambda \in \Lambda_{0}\right\}\right) \cap A \\
& =A \cap\left(X \backslash \cup\left\{U_{\lambda}: \lambda \in \Lambda_{0}\right\}\right)=A \backslash \cup\left\{U_{\lambda}: \lambda \in \Lambda_{0}\right\} \\
& =A \backslash\left(A \cap\left[\cup\left\{U_{\lambda}: \lambda \in \Lambda_{0}\right\}\right]\right)=A \backslash \cup\left\{U_{\lambda} \cap A: \lambda \in \Lambda_{0}\right\}
\end{aligned}
$$

Therefore, we have $A \backslash \cup\left\{U_{\lambda} \cap A: \lambda \in \Lambda_{0}\right\}=A \cap H \in \mathcal{H}_{A}$. This shows that $A$ is a $\mu \mathcal{H}_{\mathcal{A}}$-Lindelöf set.

The well-known result that a $\mu$-closed subspace of a $\mu$-Lindelöf space is $\mu$-Lindelöf which is a special case by taking $\mathcal{H}=\{\phi\}$.

Theorem 4.13. Let $(X, \mu, \mathcal{H})$ be a HGTS and $A \subseteq X$. If for each $\mu$-open set $U$ containing $A$ there is a $\mu_{B} \mathcal{H}_{\mathcal{B}}$-Lindelöf set $B$ with $A \subset B \subset U$, then $A$ is $\mu_{A} \mathcal{H}_{\mathcal{A}}$-Lindelöf.

Proof. Let $\left\{U_{\lambda}: \lambda \in \Lambda\right\}$ be a $\mu_{A}$-covering of $A$, where $U_{\lambda}=V_{\lambda} \cap A$ such that $V_{\lambda} \in \mu$. By the given condition, there exists a $\mu_{B} \mathcal{H}_{B}$-Lindelöf set $B$ with $A \subset B \subset \cup V_{\lambda}$. Then $\left\{V_{\lambda} \cap B: \lambda \in \Lambda\right\}$ is a $\mu_{B}$-covering of $B$. By assumption $B$ is $\mu_{B} \mathcal{H}_{B}$-Lindelöf, there exists a countable subset $\Lambda_{0}$ of $\Lambda$ such that $B \backslash \cup\left\{V_{\lambda} \cap B: \lambda \in \Lambda_{0}\right\} \in \mathcal{H}_{B}$. Let $B \backslash \cup\left\{V_{\lambda} \cap B: \lambda \in \Lambda_{0}\right\}=H \cap B$, where $H \cap B \in \mathcal{H}_{B}$ and $H \in \mathcal{H}$. Since $B=\cup\left\{V_{\lambda} \cap B: \lambda \in \Lambda_{0}\right\} \cup(H \cap B)$. Then $A \cap B=A \cap\left(\cup\left\{V_{\lambda} \cap B: \lambda \in \Lambda_{0}\right\} \cup(H \cap B)\right)=\cup\left\{V_{\lambda} \cap B \cap A\right.$ : $\left.\lambda \in \Lambda_{0}\right\} \cup(H \cap B \cap A)$. This implies $A=\cup\left\{V_{\lambda} \cap A: \lambda \in \Lambda_{0}\right\} \cup(H \cap A)$. It follows that $A \backslash \cup\left\{V_{\lambda} \cap A: \lambda \in \Lambda_{0}\right\} \subseteq H \cap A \in \mathcal{H}_{A}$. Therefore, $A$ is $\mathcal{H}_{A}$-Lindelöf.

Theorem 4.14. Every $\mu g$-closed subset of a $\mu \mathcal{H}$-Lindelöf space is $\mu \mathcal{H}$ Lindelöf relative to $X$.

Proof. Let $A$ be any $\mu g$-closed of $(X, \mu, \mathcal{H})$ and $\left\{U_{\lambda}: \lambda \in \Lambda\right\}$ be any cover of $A$ by $\mu$-open sets in $X$. Since $A$ is $\mu g$-closed, $A \subset \cup U_{\lambda}$ implies $c_{\mu}(A) \subset \cup U_{\lambda}$. Then the family $\left\{U_{\lambda}: \lambda \in \Lambda\right\} \cup\left\{X \backslash c_{\mu}(A)\right\}$ is a $\mu$ covering of $X$ and hence, there exists a countable subset $\Lambda_{0}$ of $\Lambda$ such that $X \backslash\left[\cup\left\{U_{\lambda}: \lambda \in \Lambda_{0}\right\} \cup\left(X \backslash c_{\mu}(A)\right)\right] \in \mathcal{H}$. Now, we have

$$
\begin{aligned}
X \backslash\left[\cup\left\{U_{\lambda}: \lambda \in \Lambda_{0}\right\} \cup\left(X \backslash c_{\mu}(A)\right)\right] & =\left[X \backslash \cup\left\{U_{\lambda}: \lambda \in \Lambda_{0}\right\}\right] \cap c_{\mu}(A) \\
& \supset\left[X \backslash \cup\left\{U_{\lambda}: \lambda \in \Lambda_{0}\right\}\right] \cap A \\
& =A \backslash\left[\cup\left\{U_{\lambda}: \lambda \in \Lambda_{0}\right\}\right] .
\end{aligned}
$$

Therefore, we have $A \backslash\left[\cup\left\{U_{\lambda}: \lambda \in \Lambda_{0}\right\}\right] \in \mathcal{H}$. Thus, $A$ is $\mu \mathcal{H}$-Lindelöf relative to $X$. 
A. QAHIS, H. H. ALJARRAH, T. NOIRI

\section{REFERENCES}

[1] Á. Császár, Generalized topology, generalized continuity, Acta Math. Hungar., 96 (2002), 351-357.

[2] Á. Császár, Modification of generalized topologies via hereditary classes, Acta Math. Hungar., 115.1-2 (2007), 29-36.

[3] Á. Császár, Generalized open sets in generalized topologies, Acta Math. Hungar., 106 (2005), 53-66.

[4] Á. Császár, Extremally disconneted genealized topologies, Annal Univ. Sci. Budapest., 47 (2004), 91-96.

[5] C. Carpintero, E. Rosas, M. Salas-Brown, and J. Sanabria, $\mu$-Compactness with respect to a hereditary class, Bol. Soc. Paran. Mat., 34.2 (2016), 231-236.

[6] E. Ekici, Generalized hyperconnectedness, Acta Math. Hungar., 133 (2011), 140147.

[7] E. Ekici, Generalized submaximal spaces, Acta Math. Hungar., 134 (2012), 132-138.

[8] T. Jyothis and J. Sunil, $\mu$-Compactness in generalized topological spaces, J. Adv. Stud. Top., 3.3 (2012), 18-22.

[9] Y. K. Kim and W. K. Min, On operations induced by hereditary classes on generalized topological spaces, Acta Math. Hungar., 137.1-2 (2012), 130-138.

[10] K. Kuratowski, Topologies I, Warszawa, 1933.

[11] T. Noiri and V. Popa, The unified theory of certain types of generalizations of Lindelöf spaces, Demonstr. Math., 43.1 (2010), 203-212.

[12] M. Rajamani, V. Inthumathi, and R. Ramesh, Some new generalized topologies via hereditary classes, Bol. Soc. Paran. Mat., 30.2 (2012), 71-77.

[13] B. Roy, On a type of generalized open sets, Appl. Gen. Topology, 12 (2011), 163173.

[14] M. S. Sarsak, Weak separation axioms in generalized topological spaces, Acta Math. Hungar., 131 (2011), 110-121.

[15] M. S. Sarsak, On $\mu$-compact sets in $\mu$-spaces, Questions Answers General Topology, 31 (2013), 49-57.

[16] L. E. D. Saraiva, Generalized quotient topologies, Acta Math. Hungar. 132.1-2 (2011), 168-173.

[17] A. M. Zahram, K. El-Saady, and A. Ghareeb, Modification of weak structures via hereditary classes, Appl. Math. Letters., 25 (2012), 869-872.

MSC2010: 54A05, 54A08, 54D10

Key words and phrases: Generalized topology, $m u$-Lindelöf space, hereditary class, $\mu$-covering

Department of Mathematics, Faculty of Science and Arts, Nagran UniverSity, Saudi Arabia

E-mail address: cahis82@gmail.com

Department of Mathematics, Faculty of Science, Yarmouk University, Irdid, JORDAN

E-mail address: hiamaljarah@yahoo.com

2949-1 Shiokita-cho, Hinagu, Yatsushiro-Shi, Kumamoto-ken, 869-5142 Japan

E-mail address: t.noiri@nifty.com 